\title{
NICE CONNECTING PATHS IN CONNECTED COMPONENTS OF SETS OF ALGEBRAIC ELEMENTS IN A BANACH ALGEBRA
}

\author{
Endre Makai, Jr., Budapest and Jaroslav Zemánek, Warszawa \\ Dedicated to the 90th anniversary of Professor Miroslav Fiedler, \\ from two grateful participants in mathematical olympiads \\ MTA Alfréd RÉnyi Institute of Mathematics, \\ H-1364 Budapest, Pf. 127, Hungary \\ http://www.renyi.mta.hu/〜makai \\ Institute of Mathematics, Polish Academy of Sciences, \\ 00-656 WARsaw, Śniadeckich 8, Poland \\ makai.endre@renyi.mta.hu, zemanek@impan.pl
}

\begin{abstract}
Generalizing earlier results about the set of idempotents in a Banach algebra, or of self-adjoint idempotents in a $C^{*}$-algebra, we announce constructions of nice connecting paths in the connected components of the set of elements in a Banach algebra, or of self-adjoint elements in a $C^{*}$-algebra, that satisfy a given polynomial equation, without multiple roots. In particular, we will prove that in the Banach algebra case every such non-central element lies on a complex line, all of whose points satisfy the given equation. We also formulate open questions.
\end{abstract}

2010 Mathematics Subject Classification. Primary: 46H20, Secondary: 46L05.

Key words and phrases. Banach algebras, $C^{*}$-algebras, (self-adjoint) idempotents, connected components of (self-adjoint) algebraic elements, (local) pathwise connectedness, similarities, analytic paths, polynomial paths, polygonal paths, centre of a Banach algebra, distance of connected components.

\section{INTRODUCTION}


Let $A$ be a unital complex Banach algebra. Sometimes we will assume that moreover $A$ is a $C^{*}$-algebra.

We let

$$
E(A):=\left\{a \in A \mid a^{2}=a\right\}
$$

be the set of idempotents of $A$, and

$$
S(A):=\left\{a \in A \mid a^{2}=a=a^{*}\right\}
$$

be the set of self-adjoint idempotents for the $C^{*}$-algebra case.

The connected components of $E(A)$ and of $S(A)$ have been investigated by many authors. To some of them we will refer later at the respective theorems. An ample literature is given in [AMMZ].

Let

$$
p(\lambda):=\prod_{i=1}^{n}\left(\lambda-\lambda_{i}\right)
$$

be a polynomial over $\mathbb{C}$, with all $\lambda_{i}$ 's distinct. In the $C^{*}$-algebra case, when considering self-adjoint elements, we will assume that all $\lambda_{i}$ 's are real. (In fact, if $q(\lambda):=\prod\left\{\left(\lambda-\lambda_{i}\right) \mid 1 \leq i \leq n, \lambda_{i} \in \mathbb{R}\right\}$, then $p(a)=0$ and $a=a^{*}$ imply $q(a)=0$. Thus below we could use $q(\lambda)$ rather than $p(\lambda)$.) The $\lambda_{i}$ 's are fixed throughout this paper.

We write

$$
E_{p}(A):=\{a \in A \mid p(a)=0\}
$$

and

$$
S_{p}(A):=\left\{a \in A \mid p(a)=0, a=a^{*}\right\}
$$

for the $C^{*}$-algebra case. Then $E(A)$ and $S(A)$ are special cases of $E_{p}(A)$ and $S_{p}(A)$ : namely, for $p(\lambda):=\lambda(\lambda-1)$.

We say that $\left\{e_{1}, \ldots, e_{n}\right\} \subset A$ is a partition of unity, or in the $C^{*}$-algebra case that $\left\{e_{1}, \ldots, e_{n}\right\} \subset A$ is a self-adjoint partition of unity, if

$$
\left\{\begin{array}{l}
\left\{e_{1}, \ldots, e_{n}\right\} \subset E(A), \text { or }\left\{e_{1}, \ldots, e_{n}\right\} \subset S(A), \\
\text { and } e_{i} e_{j}=0 \text { for } 1 \leq i, j \leq n \text { and } i \neq j \\
\text { and } \sum_{i=1}^{n} e_{i}=1
\end{array}\right.
$$


The detailed proofs of the statements announced in Section 2 will be published in [MZ]. The idea of this development originates from personal conversations of the authors at the conference Operator Theory and Applications: Perspectives and Challenges, held in Jurata (Hel), Poland, March 18-28, 2010, and from the 2011 lecture by the first named author [Mak].

\section{Theorems}

The "only if" part of the following Proposition 1 comes from the Riesz decomposition theorem.

Proposition 1. Let $A$ be a unital complex Banach algebra ( $C^{*}$-algebra). Let $a \in A$. Then $a \in E_{p}(A)\left(a \in S_{p}(A)\right)$ if and only if there exists a (self-adjoint) partition of unity $\left\{e_{1}, \ldots, e_{n}\right\}$ such that

$$
a=\sum_{i=1}^{n} \lambda_{i} e_{i}
$$

In the "only if" part, for $a \in E_{p}(A)$ (for $a \in S_{p}(A)$ ) one can choose the $e_{i}$ 's as polynomials of a, with complex (real) coefficients, which depend only on the $\lambda_{i}$ 's.

This representation provides the tool for reducing questions about $E_{p}(A)$ (about $S_{p}(A)$ ) to those about $E(A)$ (about $S(A)$ ). Of course, for the respective proofs for $E_{p}(A)$ (for $S_{p}(A)$ ) one has to work still substantially. As an illustration, we include a sketch of proof of Theorem 7 in Section 3.

The distinctness of the $\lambda_{i}$ 's is essential in order that $a$ should have such a simple form. For $T \in A:=B\left(l^{2} \oplus l^{2}\right)$, having a block matrix form $\left(T_{i j}\right)_{i, j=1}^{2}$, which is subdiagonal (i.e., strictly lower triangular), we have $T^{2}=0$, but $T_{21} \in B\left(l^{2}\right)$ can be as complicated as an element of $B\left(l^{2}\right)$ can be.

A path in a topological space $X$ is a continuous map $f:[0,1] \rightarrow X$. We will say that $f(0), f(1) \in X$ are connected by this path $f$. By a small abuse of language we will also say that $f([0,1]) \subset X$ is a path in $X$ (e.g., for polygonal paths). A topological space $X$ is pathwise connected if any two of its points are connected by a path in $X$. A topological space $X$ is locally pathwise connected if each point $x \in X$ has a base of (not necessarily open) neighbourhoods consisting of pathwise connected sets.

Theorem 2. Let $A$ be a unital complex Banach algebra and $C$ a connected component of $E_{p}(A)$. Then $C$ is a relatively open subset of $E_{p}(A)$. Further, $C$ is locally pathwise connected via each of the following types of paths:

1) similarity via an exponential function, i.e., $t \mapsto e^{-c t} a e^{c t}$;

2) a polynomial path of degree at most three;

3) a polygonal path of $n$ segments. 
For $E(A)$, relative openness of $C$ was proved by J. Zemánek [Ze], 1) was proved by J. Zemánek [Ze], 2) was proved by J. Esterle [Es] and M. Trémon [Tr85], 3) was proved by Z. V. Kovarik [Ko] (cf. also [Ze]).

Theorem 3. Under the hypotheses of Theorem 2, C is pathwise connected via each of the following types of paths:

1) similarity via a finite product of exponential functions, i.e., $t \mapsto e^{-c_{m} t} \ldots e^{-c_{1} t} a$ $e^{c_{1} t} \ldots e^{c_{m} t}$

2) a polynomial path;

3) a polygonal path.

In fact, there is a path satisfying 1) and 2) simultaneously.

For $E(A), 1)$ was proved by J. Zemánek [Ze], 2) was proved by J. Esterle [Es] and M. Trémon [Tr85], 3) was proved by Z. V. Kovarik [Ko] (cf. also [Ze]), and the last sentence was proved by [Es] and [Tr85].

Problem. Does there exist a uniform bound on the "minimum degree" of these polynomial connections, possibly depending on $n$, valid for all Banach algebras? Does such a bound exist, depending on $n$ and on $A$ (or even on $C$ )? Even the case of a uniform bound for polynomial connections of idempotents is open, even if we allow dependence of the bound on $A$ (or even on $C$ ). For some particular cases, see [Tr85] and [MZ89]. ([Tr95] announced a further partial result, but his proof seems to be incorrect.)

Even the "simplest" case $A:=B\left(l^{2}\right)$ is open. (The case $A=: B\left(\mathbb{C}^{n}\right)$ is solved positively by [Tr85], the uniform bound being 3, which is sharp. Here the connected components of $E(A)$ consist of the projections of the same rank.) For $A=B\left(l^{2}\right)$, the connected components of $E(A)$ are $\{e \in A \mid \operatorname{dim} N(e)=\alpha, \operatorname{dim} R(e)=\beta\}$, where $0 \leq \alpha, \beta \leq \aleph_{0}$ are cardinalities with $\alpha+\beta=\aleph_{0}$, cf. [AMMZ] $(N(\cdot)$ is the null-space and $R(\cdot)$ is the range). By [MZ89], for $\min \{\alpha, \beta\}<\aleph_{0}$, in the respective connected component there exists an at most third degree polynomial path between any two elements of that component. But even the case $\alpha=\beta=\aleph_{0}$ here is open.

Theorem 4. Let $A$ be a unital complex $C^{*}$-algebra, and $C$ a connected component of $S_{p}(A)$. Then $C$ is a relatively open subset of $S_{p}(A)$. Further, $C$ is locally pathwise connected by similarities via exponential functions, i.e., $t \mapsto e^{-i c t} a e^{i c t}$, where additionally $c=c^{*}$.

For $S(A)$, Theorem 4 was proved by S. Maeda [Mae] (cf. also [Ze]).

Theorem 5. Under the hypotheses of Theorem 4, $C$ is pathwise connected by similarities via finite products of exponential functions, i.e., $t \mapsto e^{-i c_{m} t} \ldots e^{-i c_{1} t} a e^{i c_{1} t}$ $\ldots e^{i c_{m} t}$, where additionally $c_{1}=c_{1}^{*}, \ldots, c_{m}=c_{m}^{*}$. 
For $S(A)$, Theorem 5 was proved by S. Maeda [Mae] (cf. also [Ze]).

For the $C^{*}$-algebra case, the analogues of 2) and 3) from Theorems 2 and 3 are false for $S_{p}(A)$. In fact, already the connected component of $S\left(B\left(\mathbb{C}^{2}\right)\right)$ consisting of all rank-one orthogonal projections does not contain any non-constant polynomial path. (The connected components of $S\left(B\left(\mathbb{C}^{n}\right)\right.$ ) consist of the orthogonal projections of the same rank.)

Theorem 6. Let $A$ be a unital complex Banach algebra ( $C^{*}$-algebra). Let a $\in$ $E_{p}(A)$ (let $a \in S_{p}(A)$ ). Then a belongs to the centre of $A$ if and only if its connected component in $E_{p}(A)$ (in $\left.S_{p}(A)\right)$ is $\{a\}$.

Theorem 6 for $E(A)$ was proved by J. Zemánek [Ze], for $S(A)$ by S. Maeda [Mae]. In Theorem 6 , of course, the "only if" part for $S_{p}(A)$ follows from the "only if" part for $E_{p}(A)$.

Theorem 7. Let $A$ be a unital complex Banach algebra, and $C$ a connected component of $E_{p}(A)$. If $C$ is disjoint from the centre of $A$, then any element of $C$ belongs to a complex line entirely contained in $C$. In particular, $C$ is unbounded.

For $E(A)$, Theorem 7 was proved by J. Zemánek [Ze].

In the $C^{*}$-algebra case even the entire $S_{p}(A)$ has a distance $\max \left\{\left|\lambda_{i}\right| \mid 1 \leq i \leq n\right\}$ from 0 , so the analogue of Theorem 7 for $S_{p}(A)$ is false.

Theorem 6 and Theorem 7 yield the next Corollary 8.

Corollary 8. Let $A$ be a unital complex Banach algebra. Then $E_{p}(A)$ is a union of its isolated points and of complex lines.

Theorem 9. There exists an explicit constant $c\left(\lambda_{1}, \ldots, \lambda_{n}\right)>0$ (depending on $\lambda_{1}, \ldots, \lambda_{n} \in \mathbb{R}$, and invariant under any $\operatorname{map}\left(\lambda_{1}, \ldots, \lambda_{n}\right) \mapsto\left(a+b \lambda_{1}, \ldots, a+b \lambda_{n}\right)$ with $a, b \in \mathbb{R}$ and $b \neq 0$ ) such that the following holds. If $A$ is a unital complex $C^{*}$ algebra, and $C_{1}, C_{2}$ are distinct connected components of $S_{p}(A)$, then the distance of $C_{1}$ and $C_{2}$ is at least $c\left(\lambda_{1}, \ldots, \lambda_{n}\right) \cdot \min \left\{\left|\lambda_{i}-\lambda_{j}\right| \mid 1 \leq i, j \leq n, i \neq j\right\}$.

Conjecture. Let $A$ be a unital complex Banach algebra $\left(C^{*}\right.$-algebra $)$ and $C_{1}, C_{2}$ distinct connected components of $E_{p}(A)$ (of $S_{p}(A)$ ). Then the distance of $C_{1}$ and $C_{2}$ is at least $\min \left\{\left|\lambda_{i}-\lambda_{j}\right| \mid 1 \leq i, j \leq n, i \neq j\right\}$.

For $n=2$ this conjecture is equivalent to the statement that this distance for $E_{p}(A):=E(A)$ (for $S_{p}(A):=S(A)$ ) is at least 1, which is due to J. Zemánek [Ze] (due to S. Maeda [Mae]). For $n \geq 3$ we do not even know whether this distance for the Banach algebra case is positive. 
If true, this conjecture would be sharp, for any Banach algebra: consider $\lambda_{i} \cdot 1$ and $\lambda_{j} \cdot 1$.

The Conjecture for the case of $S_{p}(A)$ would follow from the Conjecture in the case of $E_{p}(A)$. In fact, different connected components of $S_{p}(A)$ are subsets of different connected components of $E_{p}(A)$, by [BFML], Section 1, Applications, 2), also taking into consideration our Proposition 1 and Theorem 3.

\section{A PROOF}

Proof of Theorem 7 from Theorem 3 and Theorem 6. If $C$ is disjoint from the centre, then by Theorem 6 it has more than one elements. Let $a_{0} \in C$ be an arbitrary element of $C$, and let $a_{1} \in C$, with $a_{1} \neq a_{0}$. Then, by Theorem 3,3$)$, there exists a non-constant polygonal path connecting $a_{0}$ to $a_{1}$ in $C$. Its first non-constant segment (counted from $a_{0}$ ) is the graph of a non-constant polynomial of degree 1 , say of

$$
\lambda \mapsto a_{0}+b \lambda, \text { from }[0,1] \text { to } C\left(\subset E_{p}(A) \subset A\right)
$$

Hence

$$
b \neq 0 \text { and we have for all } \lambda \in[0,1] \text { identically } p\left(a_{0}+b \lambda\right)=0 .
$$

Then the equation in (1) is a polynomial equation, with coefficients from $A$ and of degree at most $n$, for $\lambda \in \mathbb{C}$. (Attention: here the coefficient of $\lambda^{n}$ is $b^{n}$, which may be 0 even for $b \neq 0$.)

We make an indirect assumption. If the polynomial

$$
\mathbb{C} \ni \lambda \mapsto p\left(a_{0}+b \lambda\right) \in A
$$

were not identically 0 for all $\lambda \in \mathbb{C}$, then for some $\lambda_{0} \in \mathbb{C}$ we would have

$$
p\left(a_{0}+b \lambda_{0}\right) \neq 0
$$

Then for some continuous linear functional $a^{\prime}$ on $A$ we would have

$$
\left\langle p\left(a_{0}+b \lambda_{0}\right), a^{\prime}\right\rangle \neq 0
$$

The polynomial

$$
\mathbb{C} \ni \lambda \mapsto\left\langle p\left(a_{0}+b \lambda\right), a^{\prime}\right\rangle \in \mathbb{C}
$$


is a $\mathbb{C}$-valued polynomial on $\mathbb{C}$ of degree at most $n$, which would not vanish at $\lambda_{0} \in \mathbb{C}$. Hence the polynomial in (3) would have at most $n$ distinct roots.

However, by (1) we have that the polynomial in (3) vanishes for all $\lambda \in[0,1]$ identically. This is a contradiction, showing that our indirect assumption is false.

That is, the polynomial in (2) is identically 0 for all $\lambda \in \mathbb{C}$. In other words, for all $\lambda \in \mathbb{C}$ we have

$$
p\left(a_{0}+b \lambda\right)=0, \text { i.e., } a_{0}+b \lambda \in E_{p}(A)
$$

which implies by connectedness of $\mathbb{C}$ that for all $\lambda \in \mathbb{C}$ we have even

$$
a_{0}+b \lambda \in C
$$

Since by (1) $b \neq 0$, we see that

$C$ contains a complex line passing through its arbitrary point $a_{0}$.

Acknowledgement. The authors are grateful to the organizers of several conferences, where this material took its shape. In particular, to the organizers of the conference Operator Theory and Applications: Perspectives and Challenges, in Jurata (Hel), Poland, March 18-28, 2010, of the 6th Linear Algebra Workshop, in Kranjska Gora, Slovenia, May 25-June 1, 2011, and of the Sz.-Nagy Centennial Conference, in Szeged, Hungary, June 24-28, 2013.

\section{REFERENCES}

[AMMZ] B. Aupetit, E. Makai, Jr., M. Mbekhta, J. Zemánek, The connected components of the idempotents in the Calkin algebra, and their liftings, In: Operator Theory and Banach Algebras, Conf. Proc., Rabat (Morocco), April 12-14, 1999 (Ed. M. Chidami, R. Curto, M. Mbekhta, F.-H. Vasilescu, J. Zemánek), Theta, Bucharest, 2003, 23-30. MR 2004g:46062.

[BFML] Z. Boulmaarouf, M. Fernandez Miranda, J.-Ph. Labrousse, An algorithmic approach to orthogonal projections and Moore-Penrose inverses, Numer. Funct. Anal. Optim. 18 (1997), 55-63. MR 97m:65105.

[Es] J. Esterle, Polynomial connections between projections in Banach algebras, Bull. London Math. Soc. 15 (1983), 253-254. MR 84g:46069.

[Ko] Z. V. Kovarik, Similarity and interpolation between projectors, Acta Sci. Math. (Szeged) 39 (1977), 341-351. MR 58\#2397.

[Mae] S. Maeda, On arcs in the space of projections of a $C^{*}$-algebra, Math. Japon. 21 (1976), 371-374. MR 56\#12900. 
[Mak] E. Makai, Jr., Algebraic elements in Banach algebras (joint work with J. Zemánek), In: 6th Linear Algebra Workshop, Kranjska Gora, Slovenia, May 25-June 1, 2011, Book of Abstracts, 26.

[MZ89] E. Makai, Jr., J. Zemánek, On polynomial connections between projections, Linear Algebra Appl. 126 (1989), 91-94. MR 91m:47026.

[MZ] E. Makai, Jr., J. Zemánek, Manuscript under preparation.

[Tr85] M. Trémon, Polynômes de degré minimum connectant deux projections dans une algèbre de Banach, Linear Algebra Appl. 64 (1985), 115-132. MR 86g:46074.

[Tr95] M. Trémon, On the degree of polynomials connecting two idempotents of a Banach algebra, Proc. Roy. Irish Acad. Sect. A 95 (1995), 233-235. MR 99f:46068.

[Ze] J. Zemánek, Idempotents in Banach algebras, Bull. London Math. Soc. 11 (1979), 177-183. MR 80h:46073. 